\section{Implementación del régimen "SERVIR" en el análisis de puestos de los servidores designados de una universidad pública peruana}

\author{
Implementation of the "SERVIR" regime in the analysis of \\ positions of designated servants of a public peruvian \\ university
}

\begin{abstract}
RESUMEN
La finalidad investigativa de este artículo es determinar la incidencia del servicio civil en el análisis de puestos del servidor público designado, tanto en el análisis situacional de los trabajadores de la universidad como en la eficiencia de los procesos de implementación. Este estudio tiene un nivel descriptivo, el método investigativo será la lógica, el instrumento que se utilizó fue revisado por los autores y fue certificada por el dictamen de expertos en el área de recursos humanos con un alfa de Cronbach que tuvo como resultado 0.93 , la muestra es de 98 servidores públicos que laboran en la UNMSM, los cuales perciben que una buena situación de un servidor en el sector educación asumirá el reto para poder adaptarse al nuevo régimen, además de la capacitación para una remuneración equitativa que permita una mayor correspondencia al trabajo, así un servidor que realiza un determinado trabajo recibe el mismo monto de pago que otro, en diferentes oficinas dentro de una misma institución. Como resultado de esta investigación se observa que conserva una influencia significativa, en términos de capacitaciones y tareas asignadas como nuevos objetivos de análisis de puestos en el nuevo régimen para el servidor estatal, que debe ser evaluado constantemente por la gestión institucional de la Autoridad Nacional del Servicio Civil (SERVIR).
\end{abstract}

Palabras claves: Servicio-Civil; Régimen-SERVIR; Análisis-Puestos; Personal-Designado.

\begin{abstract}
The investigative purpose of this article is to determine the incidence of the civil service in the analysis of positions of the designated public servant, both in the situational analysis of the university workers and in the efficiency of the implementation processes. This study has a descriptive level, the investigative method will be logical, the instrument that was used
\end{abstract}

Manuel Jesus Landa Rojas ${ }^{1}$ manuel.landarojas@gmail.com

César Astete Flor ${ }^{2}$ cesar.astete1@unmsm.edu.pe José Alberto Gamonal Montoya $^{1}$

jose.gamonal@unmsm.edu.pe

${ }^{1}$ Universidad Nacional Mayor de San Marcos, Facultad de Ciencias Administrativas. Lima, Perú ${ }^{2}$ Universidad Nacional Mayor de San Marcos, Facultad de Ciencias Económicas. Lima, Perú

Presentado: 09/02/2021 - Aceptado: 18/03/2021 - Publicado: 17/06/2021

(C) Los autores. Este artículo es publicado por la revista Gestión en el Tercer Milenio de la Facultad de Ciencias Administrativas Universidad Nacional Mayor de San Marcos. Este es un artículo de acceso abierto, distribuido bajo los términos de la licencia Creative Commons Atribución 4.0 Internacional (CC BY 4.0) [https://creativecommons.org/licenses/by/4.0/deed.es] que permite el uso, distribución y reproducción en cualquier medio, siempre que la obra original sea debidamente citada de su fuente original. 
was reviewed by the authors and was certified by the opinion of experts in the area of human resources with a Cronbach's alpha that resulted in 0.93, the The sample is of 98 public servants who work in the UNMSM, who perceive that a good situation of a servant in the education sector will assume the challenge to be able to adapt to the new regime, in addition to training for equitable remuneration that allows greater correspondence to the work, so a server who performs a certain job receives the same amount of payment as another, in different offices within the same institution. As a result of this research, it is observed that it retains a significant influence, in terms of training and tasks assigned as new job analysis objectives in the new regime for the state servant, which must be constantly evaluated by the institutional management of the National Authority of the Civil Service (SERVIR).

Keywords: Civil Service; SERVIR-Regime; Job-Analysis; Designated-Personnel.

\section{INTRODUCCIÓN}

En los procesos de reforma que se realizan en cualquier sector del Estado lleva a una reflexión sobre las complejidades del procedimiento que se da en su implementación, las alertas que se encienden en la puesta en práctica de alguna reforma se imparten sobre la necesidad de aplicar ajustes en el diseño y la pertinencia de un modelo desactualizado, en el que los actores responsables de la ejecución puedan tomar medidas que no perjudiquen a los involucrados, mientras la política en cuestión sea legítima y visible.

En este contexto, la reforma del Servicio Civil en el Perú se realiza tomando como referencia algunas acciones tomadas de otros países, que dieron como resultado a la ley SERVIR, que pretende hacer cambios en sus procesos para que un servidor sea capacitado y ubicado en el puesto que sea óptimo, siguiendo la nueva óptica de la administración de recursos humanos que es el involucramiento de las competencias del talento humano.

Este concepto es fuertemente aplaudido por Alles (2010) quien especifica que "las competencias del talento humano son características de personalidad observados en los comportamientos de cada persona y pueden generar un desempeño exitoso en un puesto de trabajo" (p. 79). Con este enfoque deberían reformar el servicio civil en todos los sectores estatales, pues es una nueva gestión de los servidores públicos. Sin embargo, hasta el 2013, el Estado no propiciaba el desarrollo de las competencias en sus servidores y se dejaba al libre albedrío su capacitación, es más hay sectores e instituciones públicos en los que hay presencia de personas que no son competentes en el puesto que lo han designado. Uno de los sectores más golpeados por esta irresponsabilidad es el sector educación (cuya autoridad es el Ministerio de Educación, en adelante MINEDU) puesto que no contaba con seminarios de capacitación, ni reglamentos que avalen el desarrollo de competencias humanas en estos servidores públicos, entre ellos están el personal de las Universidades.

SERVIR (2016) asegura que en el ámbito de la modernización del Estado peruano, se requiere una nueva reglamentación para el proceso de selección, planeamiento, ejecución, control y comunicación en el sistema administrativo actual, pasando por un riguroso análisis de cargo por competencias y selección de los servidores públicos en el área de recursos humanos de las diferentes entidades y organizaciones estatales, apuntalando la meritocracia; tal como indica la Secretaría de Gestión Pública de la Presidencia del Consejo de Ministros (2012) en un documento apoyando la nueva política de Gestión Moderna del Estado.

En este contexto, se encuentra también un problema que ha causado el desaliento de la reforma, el personal designado por la autoridad institucional, excluido de la carrera administrativa de acuerdo con lo establecido por el artículo $2^{\circ}$ de la Ley citada por Beltrán (2013), en la que dice expresamente que "no están comprendidos en la Carrera Administrativa los servidores públicos contratados ni los funcionarios 
que desempeñan cargos públicos designados o de confianza" (p. 6). Por ende, estos últimos son elegidos "a dedo" para ocupar el puesto, (claro está que el personal de las Universidades lo hace más desapercibido y con menos atención) y muchas veces este personal no es la persona idónea para el cargo designado.

Para resolver este impase en la reforma del servicio civil, sustenta la solución de este problema pasando de los perfiles de cargo por funciones al perfil de cargo por competencias. Donde el funcionario público no observará mucho los conocimientos del servidor, sino la forma cómo se desenvuelve ante un problema.

Como mencionan Tello y Astete (2020) muchos investigadores contemplan que:

el nivel de las competencias del talento humano (es decir, las enlazadas con la personalidad y el comportamiento) obtienen un vínculo muy cercano con la capacidad de hacer eficazmente las tareas del trabajo que se le asigna, por tanto, es influyente en el bienestar de la organización. (p. 32)

Por eso, el desarrollo del marco teórico se ajustó a la observación de una óptima selección del personal en cuanto al análisis situacional de los trabajadores de la Universidad y cómo evolucionar sus habilidades dentro de la organización, además, de hacer más eficiente los procesos de implementación.

\section{Modernización del Estado Peruano y Recursos Humanos}

La modernización del Estado peruano trae consigo mejoras en todo aspecto, por lo que se debe cambiar el manejo de muchas instituciones estatales que, hace un gran tiempo está en decadencia, no solo por la corrupción sino por los recursos humanos que están siendo renovados, mal remunerados y sin motivación alguna en la labor que realizan como servidor público.

En el Perú existe muchas deficiencias perceptibles en la administración pública por la ineficiencia de los funcionarios no idóneos para el cargo, que pasa por problemas de selección de personal hasta la falta de una buena política de remuneraciones, así como la falta de capacitaciones del personal que estén en el puesto indicado.
Además, las compensaciones deberían establecerse en función al perfil del personal designado.

\section{Administración del Personal y Análisis de puestos}

El Análisis de Puestos De Trabajo (APT) se define como el "proceso de identificación a través de la observación, la entrevista y el estudio, de las tareas, actividades, los factores técnicos y ambientales del puesto; así como las habilidades, conocimientos, aptitudes y responsabilidades que se requieren del trabajador" (Ivancevich, 2004).

Por otro lado, Fernández (1995) menciona que en "la Información del Análisis de Puestos" existen procedimientos para analizar y describir puestos de trabajo que nos pueden dar información diversa.

\section{Perspectiva sobre Análisis de Puestos}

Según Werther, Davis y Guzmán (2014) mencionan que:

existe un amplio conocimiento de la información sobre los puestos y requisitos y para llenarlos se obtienen a través de un proceso denominado análisis de puestos, en el cual los analistas de puestos recaban la información sobre diferentes trabajos de manera sistemática, la evalúan y organizan. Los analistas de puestos son especialistas del departamento de capital humano, cuya labor consiste en obtener datos sobre todos los puestos de trabajo que existen en la organización, pero no necesariamente sobre cada una de las personas que la componen. (p. 256)

\section{Información para el Análisis de Puestos}

Antes de hacer el estudio de los cargos es fundamental dar a conocer la información a los colaboradores de cuáles son los motivos que llevaron a la organización a realizarlo. "Tanto las razones como los resultados del trabajo se deberán dar a conocer en todos los niveles, haciendo pública una descripción específica de su función, lo que evitará desconciertos y rumores entre los empleados y garantizará su colaboración" (Werther y Davis, 2008, p. 156). En caso de que no suceda, los colaboradores podrían 
sentirse amedrentados y podrían no querer colaborar en el desarrollo al momento de obtener referencias.

Otro paso fundamental antes de obtener toda la información se basa en que el profesional se sienta familiarizado con la empresa y con todo el entorno externo. Según Werther y Davis (2008) "debe conocer a fondo el propósito, la estrategia, la estructura, los insumos (personal, materia prima y procedimientos) y los productos de la organización (tanto los objetos como los servicios que proporciona)" (p. 31).

\section{Identificación del puesto}

Antes de conseguir las referencias, los analistas, según Werther y Davis (2008) requieren "conocer cuáles son los distintos puestos que existen en la organización. Si ya se ha llevado a cabo un análisis de puestos, los analistas deben tener acceso a los registros, para identificar muchos de los puestos de la compañía" (p. 18).

\section{Desarrollo del cuestionario de análisis de puestos}

Los analistas la mayoría de las veces hacen una secuencia de formularios que ayuda a conseguir datos congruentes y homogéneos en todos los casos. Es fundamental usar formularios iguales para cargos como similitudes (Ver Figura 1).

\section{Recursos Humanos en el Perú}

Según Rivera (2013) el origen de los recursos humanos en el Perú tiene las siguientes características:

Primero, la creación de una unidad de Relaciones Industriales surgió por mandato legal y no por una iniciativa del sector privado. Luego, su creación hace referencia a un entorno donde las empresas mostraban deficiencias en la gestión de sus trabajadores y carencia de técnicas modernas. En este contexto, es el Estado el que interviene, estableciendo incluso las primeras funciones de esta unidad, las cuales son velar los derechos de los trabajadores y cumplimiento de las obligaciones de la empresa, dado el contexto de desequilibrio de poderes entre ambas partes. Por último, las características de la persona que deberá liderar la unidad, el gerente de Relaciones Industriales, también fueron definidas por ley. (pp. 216-220)

En la Tabla 1, se explica que en los últimos años se ha entendido la gestión de RR. HH. en las empresas peruanas según el trabajo realizado por Rivera (2010) quien menciona en su estudio que para comprender la evolución de recursos humanos, se tiene en cuenta los diferentes gobiernos e instancias políticas que

\section{Figura 1}

Fases de la información de análisis de puestos

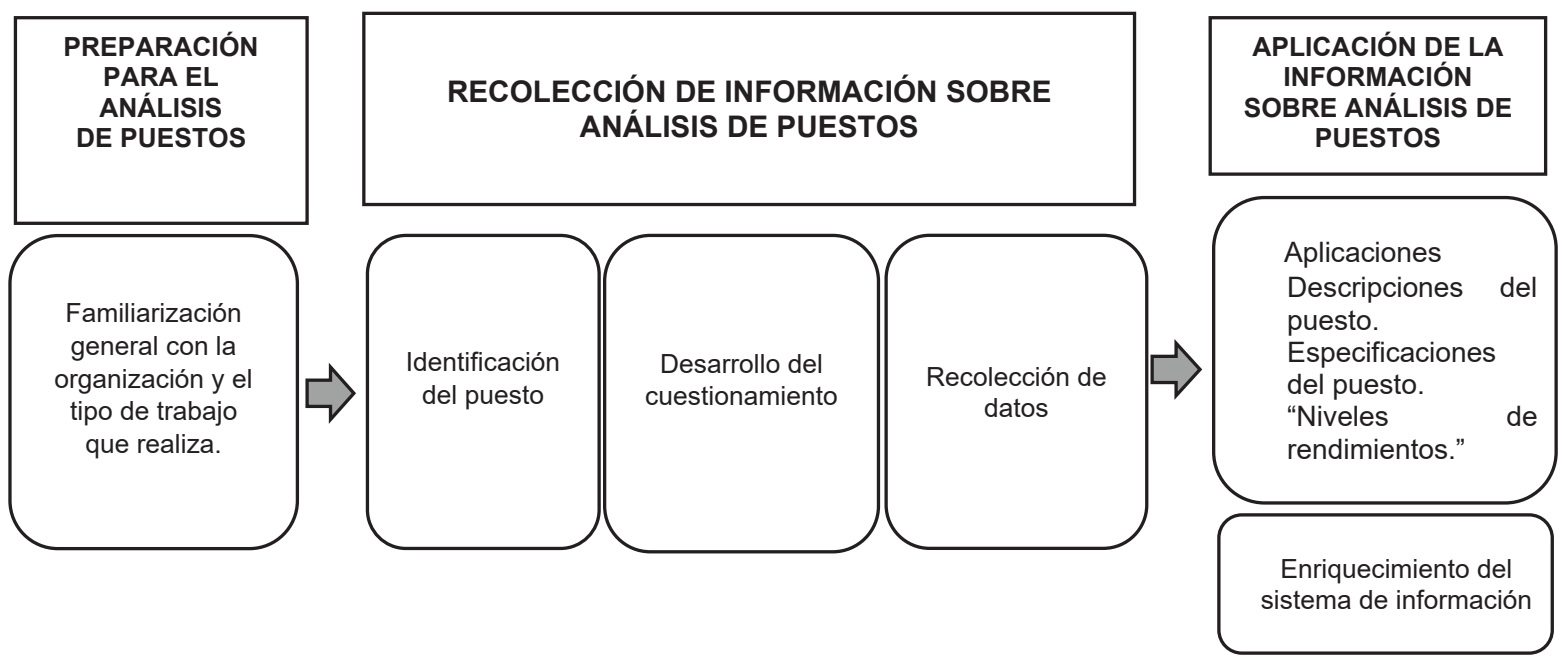


generaron diversas maneras de conceptualizar los recursos humanos, pues cada gobierno hizo sus propias leyes y reglamentos, de acuerdo a las corrientes que emanaban rápidamente aceptados por las grandes compañías.

\section{El Servicio Civil en el Perú}

Según la Autoridad Nacional del Servicio Civil (2012) la "carrera administrativa en el Perú es un conjunto de principios, normas y procesos que regulan el ingreso, los derechos y los deberes de los servidores públicos que, con carácter estable, prestan servicios de naturaleza permanente en la administración pública" (p. 20). El propósito es la integración de colaboradores oportunos, asegurando su estadía y proceso, por encima de la base de merecimiento y calificaciones en la ejecución de las labores que se requiere y en una distribución adecuada de equipos ocupacionales y de niveles.

Dicha carrera tiene a cargo 03 equipos laborales diferentes y con distintas categorías cada uno, donde la uniformidad de remuneración está fijada mediante un método exclusivo de retribuciones.

Además, la Ley registra como servidor público a las personas que dan servicios en instituciones de la gestión pública con nominación o compromiso de trabajo, con todos los requisitos de ley, en acto legítimo y dispuesto a remuneraciones en etapas regulares. Así mismo, la Ley establece que solo realiza carrera administrativa al trabajador público con nombramiento que da servicios de manera constante, y al mismo tiempo tiene solidez en el trabajo indefinido.

Longo (2006) propone como guía, acerca del método de administración de trabajo y de los recursos humanos, con afán de formar algo relacionado que ayude a dar diagnósticos, y futuras evaluaciones. Además, la Directiva $N^{\circ} 002-2014-$ SERVIR/GDSRH, que tiene como finalidad "proporcionar los lineamientos a seguir por las entidades públicas en lo concerniente a sus procesos vinculados al sistema administrativo de gestión de recursos humanos" (pp. 426-431).

\section{Procesos de gestión de servidores}

La Autoridad Nacional del Servicio Civil (2012) indica que "la gestión de servidores bajo el régimen laboral público administrativo tiene ocho procesos identificados" (p. 25), tal como se puede observar en la Figura 2:

Entre los más destacados para los resultados de este artículo se encuentran:

\section{Selección.}

Guth (2001) menciona que la selección de personal es "el proceso mediante donde la empresa

Tabla 1

Evolución de la Gestión de Recursos Humanos en el Perú

\begin{tabular}{|c|c|c|c|c|c|}
\hline Periodo & $\begin{array}{l}\text { Situación del } \\
\text { Perú }\end{array}$ & Denominación & Reporta a & Plan de RR. HH & Tema principal \\
\hline $1970-1980$ & Dictadura militar & $\begin{array}{l}\text { Relaciones } \\
\text { industriales }\end{array}$ & Operaciones & \multirow{3}{*}{$\begin{array}{l}\text { Propio y basado en } \\
\text { temas de moda }\end{array}$} & $\begin{array}{c}\text { Servicio de relaciones industriales, } \\
\text { derechos laborales de los trabajadores } \\
\text { de las industrias }\end{array}$ \\
\hline $1980-1990$ & Hiperinflación & $\begin{array}{l}\text { Administración de } \\
\text { Personal }\end{array}$ & Finanzas & & $\begin{array}{c}\text { Estabilidad laboral absoluta: } \\
\text { incentivo perverso a la ineficiencia } \\
\text { y la mediocridad }\end{array}$ \\
\hline $1990-2000$ & Terrorismo & $\begin{array}{l}\text { Gestión de Recursos } \\
\text { Humanos }\end{array}$ & Administración & & $\begin{array}{l}\text { Gestión y eliminación de sindicatos, } \\
\text { tercerización de actividades }\end{array}$ \\
\hline 2000-2010 & $\begin{array}{l}\text { Estabilidad } \\
\text { Política }\end{array}$ & Gestión de Personas & $\begin{array}{l}\text { Gerencia Central } \\
\text { Vicepresidencia } \\
\text { Gerencia General }\end{array}$ & \multirow{2}{*}{$\begin{array}{l}\text { Alineado con el plan } \\
\text { de la empresa y } \\
\text { específico para esta }\end{array}$} & $\begin{array}{c}\text { Alinearse con la estrategia del } \\
\text { negocio y acompañar a la empresa en } \\
\text { su crecimiento planes a medida de la } \\
\text { empresa }\end{array}$ \\
\hline 2010-Hoy & $\begin{array}{l}\text { Crecimiento } \\
\text { Económico }\end{array}$ & $\begin{array}{l}\text { Gestión y Desarrollo } \\
\text { Humano }\end{array}$ & Gerencia General & & $\begin{array}{l}\text { Escasez de talento, prohibición de } \\
\text { despido arbitrario y defensa de } \\
\text { derechos fundamentales }\end{array}$ \\
\hline
\end{tabular}

Nota. Adaptado de De relaciones industriales a gestión y desarrollo humano por M. Rivera, 2013, p. 11. 
Figura 2

Procesos de gestión de servidores

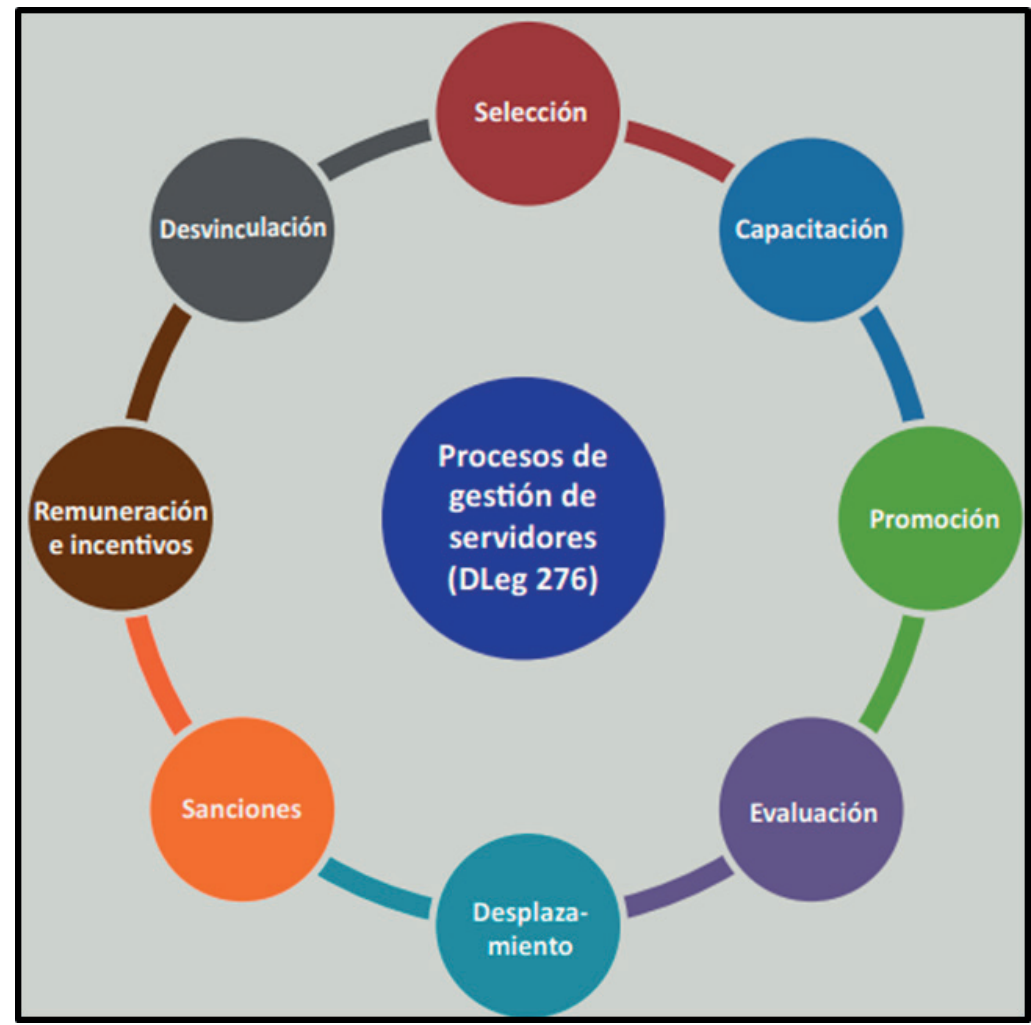

Nota. Adaptado de El Servicio Civil peruano, por la Autoridad Nacional del Servicio Civil, 2012, p. 25.

elije a la persona más adecuada y que cumpla con los requisitos establecidos para desempeñar el cargo vacante" (p. 24).

\section{Capacitación.}

La capacitación según Salinas (2012) es un "proceso en el que se alimenta a la organización de recursos humanos, ya que del éxito de la ejecución de este proceso dependerá de las necesidades de nuevo personal que responda a los requerimientos técnicos de sus unidades productivas o administrativas".

\section{Promoción.}

Una promoción resulta cuando "se cambia a un empleado a una posición de mejor nivel de compensación, con mayores responsabilidades y a un nivel corporativo más alto". (Werther y Davis, 2008, p. 239)

\section{Compensaciones.}

La compensación, según Osterling (2012) es "un modo de extinción de las obligaciones que opera cuando dos personas son respectivamente acreedoras y deudoras una de la otra" (p. 23). En virtud de la compensación las dos relaciones obligatorias se extinguen recíprocamente, hasta donde alcance el importe de la menor de ellas

\section{Remuneraciones e incentivos.}

Conforme con la Ley de bases de la carrera administrativa, "para efectos de la carrera administrativa y sistema único de remuneraciones, la administración pública constituye una sola institución, sin perjuicio del nivel de gobierno al que pertenezca un servidor en particular o a la estructura organizacional de cada entidad" (p.230).

\section{¿Qué funciones desempeñan los servido- res públicos?}

La Escuela de Gobierno y Gestión Pública (2016) nos indica las funciones que ejerce cada servidor por rango o nivel de jerarquía, así se muestra: 


\section{Funcionarios y directivos}

Tiene las funciones de conducción y dirección como alcaldes y jefes de entidades.

\section{Profesionales}

Asumen el reto de desarrollar políticas públicas y hacer que se ejecuten los servicios públicos, cuentan con estudios superiores.

\section{Técnicos}

Son de apoyo administrativo, tiene estudios técnicos.

\section{Auxiliares}

Realizan actividades complementarias como mantenimiento, limpieza, seguridad, conducción de vehículos, obras de construcción, etc. Solo se requiere una educación básica.

\section{Carreras especiales}

Realizan funciones específicas en Ministerios como educación, salud, justicia, defensa, etc.

\section{MÉTODOS}

Este estudio tiene un nivel descriptivo, ya que, se analiza una cuestión mediante un instrumento de medición de datos argumentando acertadamente una posible respuesta. El método de investigación, conforme con Sierra (2001) será la lógica, ya que "los conceptos y enunciados surgen de una revisión y sistematización presentada en el marco teórico; a partir del cual se realiza la contrastación empírica en una realidad concreta" (p. 21).

Por otro lado, Arias y Peñaloza (2013) explican que "estadísticamente la adjetivación de población finita o va a depender de la infinita posibilidad del investigador de contar con un listado completo de los individuos, sujetos $\mathrm{u}$ objetos investigados" (p. 9). Por ello se ha establecido que la unidad de esta investigación es el trabajador (servidor) designado de una Universidad pública, en este caso es la Universidad Nacional Mayor de San Marcos (UNMSM), donde la población estimada es 132 servidores públicos designados, los cuales se analizarán en el presente artículo. Sustituyendo en la fórmula de Fisher y Navarro (1996) se consigue un tamaño de muestra (n) de 98 trabajadores, los cuales se elegirán manera aleatoria para que respondan una encuesta que servirá de guía para la obtención de resultados de esta investigación.

El instrumento que se utilizó fue revisado por los autores y fue certificada por el dictamen de expertos en el área de recursos humanos, igualmente se validó su buena credibilidad en consistencia interna "a través de alfa de Cronbach que tuvo como resultado 0.93 , lo cual revela que el instrumento es replicable y los resultados que hay en este estudio son válidos" (p. 268).

Cabe recalcar "que el instrumento tuvo las preguntas que miden los indicadores, los cuales brindan la percepción de" 98 servidores públicos que laboran en la UNMSM, sobre la implementación del régimen SERVIR (primera variable) que se tomará de acuerdo a la meritocracia y calificaciones en el ejercicio de sus labores, considerando una organización uniforme de grupos ocupacionales y de niveles; además, entender con precisión su relación $\left(\mathrm{X}-\mathrm{y}_{1} ; \mathrm{X}-\mathrm{y}_{2}\right)$ con las dimensiones del análisis de puestos (segunda variable) cuyas dimensiones se observa en la Tabla 2. Así, se consideran algunas preguntas de apoyo para la primera variable son dadas para las dimensiones competencias del personal y tareas asignadas que son numeradas del

Tabla 2

Distribución de dimensiones

\begin{tabular}{cccc}
\hline Variables & Dimensiones & Tipo de Ítem & Ítems \\
\hline Implementación del Régimen & Competencias $\left(\mathrm{x}_{1}\right)$ & Escala de Likert & 1 al 3 \\
SERVIR $(X)$ & Tareas asignadas $\left(\mathrm{x}_{2}\right)$ & Escala de Likert & 4 al 7 \\
Análisis de puestos $(\mathrm{Y})$ & T, implementado $\left(\mathrm{y}_{1}\right)$ & Escala de Likert & 20 al 22 \\
& Análisis Situacional $\left(\mathrm{y}_{2}\right)$ & Escala de Likert & 23 al 25 \\
\hline
\end{tabular}

Nota. Elaboración propia. 
1 al 7 y, las dimensiones tiempo de Implementación, análisis situacional de los trabajadores de la Universidad., eficiencia de los procesos de implementación, aplicación de la mejora interna en la segunda variable son del 20 al 30. Cada Ítems de la encuesta posee 5 alternativas en escala Likert, cuyos puntajes son como sigue: $1 \mathrm{p}$. totalmente en desacuerdo; $2 \mathrm{p}$. en desacuerdo; $3 p$. indiferente; $4 p$. de acuerdo, $5 p$. totalmente de acuerdo. cuyos resultados se exponen en la sección "Resultados" de esta investigación.

\section{RESULTADOS}

A continuación, se comenta una relación entre las dimensiones de las dos variables, cuyos resultados son expuestos aplicando la encuesta (instrumento). Así se observa que en la Tabla 3 la relación entre la implementación del Régimen SERVIR sobre las dimensiones del análisis de puestos que son tiempo de Implementación y análisis situacional es positiva, es decir, si la implementación del nuevo Régimen laboral de los servidores públicos mejora la situación actual, el análisis de puestos también lo hará.

\section{Resultado de la Relación Específica 1}

Regresión de la Dimensión Tiempo de Implementación sobre la variable Régimen de Servicio Civil

Se propone la siguiente ecuación del Modelo Lineal:

$$
T I_{i}=\beta_{0}+\beta_{1} R S C+u_{i}
$$

Donde:

TI: Mide la dimensión Tiempo de Implementación.
RSC: Mide la variable Régimen de Servicio Civil

i: Observación del entrevistado número "i" ("i" de 1 a 98).

Los resultados obtenidos en la regresión son los siguientes:

La presente muestra desprende un coeficiente $\beta 1$ que tiene tendencia positiva, es decir, cuando incrementa la RSC en un punto, también lo hace el Tiempo de Implementación 0.77 puntos. Por otro lado, el error estándar es de $8 \%$. También se precisa que el 49\% (1- $R^{2}$ )" de los cambios en el Tiempo de Implementación es explicado por otros factores distintos al Régimen Servicio Civil.

Ho: $\boldsymbol{\beta 1}=\mathbf{0}$ (El Régimen del Servicio Civil no tiene un impacto sobre el Tiempo de Implementación)

H1: $\boldsymbol{\beta 1} \neq \mathbf{0}$ (El Régimen del Servicio Civil tiene un impacto sobre el Tiempo de Implementación)

Se obtiene un T- Statistic de 9.89 (Fuera de la zona de aceptación) y superior al $\mathrm{T}$ - crítico: 1.96 y un P-valor menor a 0.05. Así, se concluye que $\beta 1$ es estadísticamente significativo.

\section{Interpretación}

La implementación del nuevo régimen SERVIR se canaliza por una serie de etapas que va a requerir de un tiempo prudente para que los servidores del sector educación puedan capacitarse y afianzar sus competencias humanas para ver qué puesto (cargo) desempañaría mejor su

Tabla 3

Resultados de la Regresión Tiempo de Implementación - Régimen de Servicio Civil

\begin{tabular}{|c|c|c|c|c|}
\hline \multicolumn{5}{|c|}{ Dependent Variable: T_Implementación } \\
\hline \multicolumn{5}{|c|}{ Method: Least Squares } \\
\hline \multicolumn{5}{|c|}{ Included observations: 98} \\
\hline Variable & Coefficient & Std. Error & $\mathrm{t}$-Statistic & Prob. \\
\hline LEY_SERVIR & 0.770994 & 0.077183 & 9.98916 & 0 \\
\hline C & 0.783252 & 0.184195 & 4.252301 & 0 \\
\hline R-squared & & & & \\
\hline
\end{tabular}

Nota. Elaboración propia. 
trabajo. Así se puede determinar que el tiempo en que se determine la implementación mejora la puesta en marcha del nuevo Régimen SERVIR en el sector educación.

\section{Resultado de la Relación Específica 2}

Regresión de la Dimensión Análisis Situacional sobre la variable Régimen del Servicio Civil

Se propone el siguiente Modelo Lineal y la Tabla 4:

$$
A S_{i}=\beta_{0}+\beta_{1} R S C_{i}+u_{i}
$$

Donde:

AS: Mide la dimensión Análisis Situacional.

RSC: Mide la variable Régimen del servicio civil.

i: Observación del entrevistado número "i" (“i” de 1 a 98).

La presente muestra desprende un coeficiente $\beta 1$ que tiene una tendencia positiva, es decir, cuando incrementa la variable RSC en un punto, incrementa el nivel de Análisis Situacional en 0.78 puntos. Por otro lado, el error estándar es 9,7\%. También se precisa que el $60 \%\left(1-R^{2}\right)$ " de los cambios en el Análisis Situacional es interpretado por otros factores distintos al régimen del servicio civil.

Ho: $\boldsymbol{\beta 1}=\mathbf{0}$ (Régimen del servicio civil no tiene un impacto sobre el Análisis Situacional)
H1: $\boldsymbol{\beta 1} \neq \mathbf{0}$ (Régimen del servicio civil tiene un impacto sobre el Análisis Situacional)

Se obtiene un T- Statistic de 8.08 (Fuera de la zona de aceptación) y superior al $\mathrm{T}$ - crítico: 1.96 y un P-valor menor a 0.05. Así, se concluye que $\beta 1$ es estadísticamente significativo.

\section{Interpretación}

La implementación del nuevo régimen SERVIR dará lugar a una serie de cambios y metas que va a requerir un análisis situacional para que los gestores del sector educación puedan conocer y gestionar las competencias humanas que debe tener cada puesto (cargo) para calcular y homogenizar el salario de los servidores del sector educación. Así se puede comprobar que el análisis situacional puede mejorar la implementación del nuevo Régimen SERVIR en el sector educación.

\section{DISCUSIÓN}

En la investigación de Toranzo (2008) se concluye que "la reforma del servicio civil va a contemplar el ordenamiento que necesitaba el estado para asegurar el buen servicio en los gobiernos locales, implementándose políticas de gestión en los servidores públicos" (p. 64). Así, en los resultados que se obtuvo debe haber un tiempo prudente para ser implementado, porque se tiene que respetar los procesos de gestión que hay en toda organización.

Además, se coincide con Vásquez (2018) pues existen muchas ambigüedades en las tareas que hace un servidor similar al de otro, pero uno de ellos tiene menos carga laboral y

Tabla 4

Resultados de la Regresión Análisis Situacional - Régimen del servicio civil.

\begin{tabular}{|c|c|c|c|c|}
\hline \multicolumn{5}{|c|}{ Dependent Variable: ANÁLISIS SITUACIONAL } \\
\hline \multicolumn{5}{|c|}{ Method: Least Squares } \\
\hline \multicolumn{5}{|c|}{ Included observations: 98} \\
\hline Variable & Coefficient & Std. Error & t-Statistic & Prob. \\
\hline LEY_SERVIR & 0.783632 & 0.097038 & 8.07550 & 0 \\
\hline $\mathrm{C}$ & 0.785888 & 0.231579 & 3.393614 & 0.001 \\
\hline R-squared & & & & \\
\hline
\end{tabular}

Nota. Elaboración propia. 
tiene mejor sueldo. Esto se da, mayormente, por la existencia de los diferentes regímenes laborales del Estado Peruano, se mantendrían en vigencia junto a la LSC siendo un régimen más y no como régimen único y exclusivo que contempla como objeto principal la Ley 30057.

Según Castro (2018) menciona que "en toda relación laboral el ejercicio del poder de dirección es propia e intrínseca al empleador y con él la facultad disciplinaria sobre todos sus trabajadores" (p. 75). Por eso se debe considerar que el hecho de no tener una amonestación, sobre todo en los servidores nombrados colabora con la indisciplina y llega a entorpecer todo acto de mejora que pueda tener una reforma laboral. Además, Corrales (2017) aduce que se debe contar con un servicio civil profesional, condición no suficiente pero necesaria para el desarrollo de los países, pues es una tarea que el estado garantice la buena administración pública que conlleva a un beneficio mayor para su población.

Por otro lado, Cánepa y Ocampos (2017) manifiestan que "la Ley del Servicio Civil a través de las facilidades otorgadas para su incorporación, la gestión del rendimiento, la gestión de la capacitación y las mejoras en las compensaciones han logrado incidir de manera positiva en la motivación laboral" (p. 83), en los servidores de Tumbes, existiendo confianza en su implementación. Lo que al contrario de los servidores en el sector educación, aún falta establecer criterios para su buena expectativa y homologación de Compensaciones.

Para Bocanegra (2017) muestran una diferencia marcada en cuanto a la implantación de la "Ley Servir entre los servidores, siendo los trabajadores del Contrato Administrativo de Servicios (CAS) y los del Decreto Legislativo 728 quienes la requieren; a su vez existe una resistencia al cambio de parte de los empleados del DL 276" (p. 9). Es así como el 33\% de servidores no están de acuerdo con este tipo de reforma, si se le quita ciertos beneficios.

\section{CONCLUSIONES}

- La implementación del nuevo régimen del servicio civil influye significativamente en el análisis de puestos, pues una vez que se tiene los perfiles adecuados para cada cargo. se tiene que capacitar al personal designado para evaluarlos, hasta ahora no todos los servidores públicos son nombrados a puestos que estén dando con el perfil adecuado, a pesar de que son cargos de confianza, todo se entrelaza en un grupo de servidores.

- El $67 \%$ de los servidores indican que están en desacuerdo que la Universidad en referencia sobre cómo evaluará la institución laboral con la implementación de la Ley SERVIR, es decir, hay una incertidumbre sobre cómo debe desempeñarse el personal en esta nueva situación laboral, y en qué tiempo se va a consolidar.

- $\quad$ El 72\% de los servidores públicos que laboran en la Universidad referente, según la encuesta realizada, cree que debe ser mejor compensado, pero el Estado no ha tomado mucha importancia a sus recursos humanos y que recién se ejecuta algunas medidas para una mejor calidad en el área de trabajo en el Estado.

\section{REFERENCIAS BIBLIOGRÁFICAS}

Alles, M. (2010). Dirección Estratégica de Recursos Humanos, Gestión por competencias. Ediciones Garnica.

Arias L., S. y Peñaloza, M. (2013). Muestreo enfoque ilustrado para investigar. Mérida: Talleres Gráficos de la Universidad de Los Andes.

Autoridad Nacional del Servicio Civil. (Mayo de 2012). El Servicio Civil Peruano. Obtenido de servir.gob.pe: http://storage.servir.gob.pe/biblioteca/SERVIR\%20-\%20E1\%20servicio\%20 civil\%20peruano.PDF

Beltrán L., L. P. (2013). Problemática de la existencia de distintos regímenes de contratación del personal en el estado. [Tesis para optar el grado de Maestría en Derecho de la Empresa con Mención en Gestión Empresarial]. Universidad Pontificia Católica del Perú. Obtenido de http://tesis.pucp.edu.pe/repositorio/handle/20.500.12404/4508

Bocanegra, W. (2017). La incidencia de la Ley del Servicio Civil en los procesos del Recurso Humano en la Unidad de Gestión Educativa Local Sánchez Carrión en el año 2016. [Tesis para el título de Licenciado en Administración]. Universidad 
nacional de Trujillo. Obtenido de https://dspace.unitru.edu.pe/handle/UNITRU/10554

Canepa Maceda, C. y Ocampos Rujel, F. H. (2017). Ley del Servicio Civil y la Motivación Laboral en el Proyecto Especial Binacional Puyango Tumbes, 2016. [Tesis para optar al Título Profesional de Licenciado en Administración]. Universidad Nacional de Tumbes. Obtenido de http://repositorio.untumbes.edu.pe/handle/UNITUMBES/91

Castro Miranda, J. H. (2018). Gestión de los Procesos Administrativos Disciplinarios en la Ley 30057 Ley del Servicio Civil - Hospital Vitarte. [Tesis para optar el Grado Académico de Maestro en Gestión Pública]. Escuela de Posgrado Universidad Cesar Vallejo. Obtenido de: https://repositorio.ucv.edu.pe/handle/20.500.12692/16111

Chiavenato, I. (2002). Administración, Teoría, Proceso y Práctica. México: Ed. Mc Graw Hill.

Chiavenato, I. y Mascaró, P. (2007). Administración de Recursos Humanos (8va. ed.). México: Mc Graw Hill. doi: ISBN: 978-970-10-6104-6

Corrales Angulo, A. M. (2017). Análisis y propuesta para la implementación del modelo de directivos públicos de la ley del servicio civil 2018 - 2020. [Tesis para optar maestría en gerencia publica]: Universidad Continental. Obtenido de https://repositorio.continental.edu.pe/handle/20.500.12394/4240?locale=ja

Dolan, S., Valle C., R., Jackson, S. y Schuller, R. (2007). La Gestión de los Recursos Humanos (3ra. ed.). Madrid: Mc Graw Hill / Interamericana de España.

Fernández R., M. (1995). Análisis y descripción de puestos de trabajo. Madrid: Ediciones Díaz de Santos.

Fisher, L. y Navarro, A. (1996). Introducción a la Investigación ( $3^{\circ}$ ed.). México: McGraw Hill.

Ivancevich, J. (2004). Administración de Recursos Humanos (9th ed.). México: Mc Graw-Hill / Interamericana. ISBN: 9701045971

Longo, F. (2006). Marco analítico para el diagnóstico institucional de Sistemas de Servicio Civil. En K. Echevarría, Informe sobre la situación del Servicio Civil en América Latina (págs. 421446). Washington DC: Banco Interamericano de Desarrollo.

Mandino, O. (1988). El vendedor más grande del mundo. México D. F.: Editorial Planeta Mexicana SA.
Presidencia del Consejo de Ministros. (2012). Política Nacional de Modernización de la Gestión Pública al 2021. Gobierno del Perú. Obtenido de http://www.pcm.gob.pe/wp-content/ uploads/2013/05/PNMGP.pdf

Reeve, J. (2020). Motivación y emoción. McGraw-Hill Interamericana.

Rivera Chú, M. (2013). De relaciones industriales a gestión y desarrollo humano. La evolución de la gestión de recursos humanos en el Perú. Obtenido de Consultora en Gestión y Desarrollo Humano: https://www.esan.edu.pe/ publicaciones/2013/06/11/tiempo_de_opinion_makaly_rivera.pdf

Sánchez J. C. y Amelia (2016). El sistema de Administración de Recursos Humanos y el Subsistema de Aprovisionamiento aplicado a las empresas de los departamentos de Matagalpa y Jinotega, año 2015.

SERVIR. (2016). Características del Servicio Civil Peruano. Lima: SERVIR.

Sierra Bravo, R. (2001). Técnicas de Investigación Social. Teoría y Ejercicios. Madrid: Paraninfo, Thomson Learning.

Tello, J. y Astete, C. (2020). Las competencias del talento humano de los martilleros en la eficiencia de las subastas en Perú. Gestión En El Tercer Milenio, 23(46), 31-40. doi: https://doi. org/10.15381/gtm.v23i46.19130

Toranzo C., A. (2008). El servicio civil y su problemática en los servidores del decreto legislativo № 276 de la Municipalidad Distrital de la Punta, Callao 2016. Tesis para optar por el Grado de Licenciado en Administración. Lima: Universidad Las Américas.

Vásquez C., H. (2018). Ley del servicio civil y su implicancia en la estabilidad laboral de los trabajadores de la GERESA Lambayeque. Perú, 2017. Para optar el título profesional de abogado. Universidad de Señor de Sipán.

Werther, W. y Davis, K. (2008). Administración del Personal Y Recursos Humanos. USA: Mc Graw Hill.

Werther, W., Davis, K. y Guzmán, M. (2014). Administración de recursos humanos: gestión del capital humano. McGraw Hill. 
